Service social

\title{
Les Québécois devant les services sociaux. Un univers de transition?
}

\section{Sylvie Jutras et Marc Renaud}

Volume 39, numéro 2, 1990

Les problèmes sociaux

URI : https://id.erudit.org/iderudit/706480ar

DOI : https://doi.org/10.7202/706480ar

Aller au sommaire du numéro

Éditeur(s)

École de service social de l'Université Laval

ISSN

1708-1734 (numérique)

Découvrir la revue

Citer cet article

Jutras, S. \& Renaud, M. (1990). Les Québécois devant les services sociaux. Un univers de transition? Service social, 39(2), 115-128.

https://doi.org/10.7202/706480ar
Résumé de l'article

Ce texte présente les résultats d'un vaste sondage d'opinions concernant des attitudes générales en matière de problèmes sociaux ou sociosanitaires et plus particulièrement par rapport à deux situations problèmes : devoir prendre en charge un parent âgé en perte d'autonomie ou devoir assumer les

responsabilités de chef de famille monoparentale. Mais en plus de l'analyse de ces deux problèmes spécifiques, cette enquête nous fournit de précieuses informations sur les types de solutions concrètes qu'une population se donne pour faire face aux divers problèmes sociaux qui la confrontent. 
Sylvie Jutras, chercheuse adjointe, Département de psychologie de I'Université de Montréal. Membre du Groupe de recherche sur les aspects sociaux de la prévention (GRASP).

Marc Renaud, professeur, Département de sociologie de l'Université de Montréal. Membre du Groupe de recherche sur les aspects sociaux de la prévention (GRASP).

\section{Les Québécois devant les services sociaux Un univers en transition ?*}

Le temps des changements semble venu en matière de services de santé et de services sociaux assurés par le réseau public. C'est du moins ce qui ressort des débats entourant la réforme des services sociaux et de santé au Québec. Un jalon majeur de cette réforme fut la Commission d'enquête sur les services de santé et les services sociaux (Commission Rochon). Cette dernière avait mis sur pied un programme de recherche pour l'aider à asseoir et orienter son analyse et ses recommandations. En particulier, elle voulait savoir de quelle façon, en 1987, les Québécois tentaient de régler leurs problèmes sociaux et de santé, en insistant sur ceux qui ne sont pas complètement pris en charge par l'univers médico-hospitalier.

$\mathrm{C}^{\prime}$ est dans ce contexte que se situe la présente étude. Nous voulions établir des modèles de recours aux solutions diverses qu'offrent le secteur public, le secteur privé, le réseau communautaire, l'individu et la famille. Nous avons étudié certains comportements de recours et opinions de la population générale et sondé plus en détail trois situations cibles : un problème sanitaire (souffrir d'un mal de dos chronique), un problème sociosanitaire (devoir prendre en charge un parent âgé en perte d'autonomie) et un problème social (assumer les responsabilités de chef de famille monoparentale). Malgré leurs limites, les données descriptives recueillies apparaissent particulièrement intéressantes dans un contexte où peu d'études panquébécoises sont réalisées auprès d'échan- 
tillons représentatifs, même si les enjeux soulevés sont très importants pour l'avenir des services sociaux.

Le présent article porte sur les principaux résultats des analyses touchant les considérations d'ordre social dans notre enquête : les données recueillies auprès de l'ensemble des 2114 répondants concernant des attitudes générales en matière de problèmes sociaux ou sociosanitaires, et auprès des échantillons spécifiques confrontés à la situation de devoir s'occuper d'un parent âgé en perte $d^{\prime}$ autonomie ou de devoir assumer seul (homme ou femme) les responsabilités de chef de famille. Notre objectif est de décrire le rapport qu'entretient la population échantillonnée avec les services sociaux, tel qu'il ressort de notre enquête, pour ensuite soulever quelques interrogations sur l'évolution des services sociaux dans le cadre de la réforme proposée.

\section{Méthodologie ${ }^{1}$}

Les données ont été recueillies par entrevue téléphonique (de 20 à 30 minutes) auprès d'un échantillon représentatif de l'ensemble des Québécois. La population visée par le sondage était celle des 18 ans et plus résidant au Québec et pouvant s'exprimer en français ou en anglais. Un modèle d'échantillonnage stratifié et non proportionnel a permis d'atteindre les quotas attendus pour chacune des situations cibles, et de décrire le plus exactement possible la réalité vécue dans certaines régions du Québec. Divers critères ont permis de dépister au téléphone les individus admissibles dans chacune des situations étudiées.

Notre échantillon de répondants comprend un peu plus de femmes que d'hommes $(53,5 \% / 46,5 \%)$. L'âge moyen des répondants est de 39,5 ans. Comme dans la population générale, les francophones sont majoritaires au sein de notre échantillon $(86,8 \%)$. Les répondants ont en moyenne 12,1 années de scolarité et ont eu, en 1986, un revenu familial moyen de $31675 \$$. Sont sur le marché du travail $79,7 \%$ des hommes et $51,6 \%$ des femmes.

\section{Attitudes et comportements de la population générale}

Cette section rapporte les principaux résultats concernant la consommation de services, la perception du système et les services produits par la population. Rappelons que l'ensemble des répondants $(n=2114)$ ont été interrogés sur ces questions. 


\section{Consommation de services}

Les Québécois sont nombreux à utiliser les services de santé du réseau institutionnel : $72,8 \%$ déclarent y avoir eu recours pendant l'année précédant l'enquête, que ce soit pour eux ou pour un proche. Par contre, les services sociaux n'ont été utilisés que par 2,9\% de la population. Au cours de l'année précédant l'enquête, près de $20 \%$ des répondants ont fréquenté un centre local de services communautaires (CLSC), 33,6 \% une urgence d'hôpital, 31,8 \% une clinique d'hôpital et $2,4 \%$ un centre de services sociaux (CSS), que ce soit pour eux ou un de leurs enfants. La demande d'aide se porte d'abord vers l'omnipraticien : $26 \%$ de l'ensemble des répondants (et plus de la moitié de ceux qui se sont sentis malades) affirment avoir consulté un omnipraticien au cours du dernier mois. En second lieu vient le médecin spécialiste $(13,0 \%)$, puis le physiothérapeute $(1,4 \%)$ et, enfin, le travailleur social $(1,0 \%)$.

Grandmaison (1981) a montré que la clientèle principale du Centre de services sociaux du Montréal métropolitain (CSSMM) provient de milieux défavorisés ( $79 \%$ ). Nos données coïncident avec ce résultat. Les répondants dont le revenu familial est inférieur à $20000 \$$ représentent $59,9 \%$ de ceux qui disent avoir fréquenté le CSS durant l'année. Dans la clientèle déclarée, on retrouve un peu plus d'individus ayant 12 ans ou moins de scolarité $(53,4 \%)$ que d'individus plus scolarisés $(46,6 \%)$. D'après Grandmaison, les femmes constituent $56 \%$ de la clientèle du CSSMM; le même chiffre est observé au niveau de tout le Québec : 56,9 \% des personnes ayant fréquenté un CSS sont des femmes. En ce qui concerne l'âge des usagers des CSS, 5,2\% des individus âgés de 18 à 24 ans ont fréquenté un CSS, alors que cette proportion varie de $0,3 \%$ (les 65 ans et plus) à $3,3 \%$ (35-44 ans) dans les autres catégories d'âge. Ces taux sont basés sur un petit nombre d'usagers de CSS $(n=51)$, mais dénotent tout de même l'importance que pourraient revêtir les services sociaux dans les premières années de la vie adulte.

Près de $8 \%$ des répondants déclarent avoir déjà consulté "quelqu'un qui aide les gens au plan psychologique ». La personne aidante est plus souvent un psychologue $(60,6 \%)$, suivi du psychiatre $(23,1 \%)$, du travailleur social $(10,5 \%)$ et du psychanalyste $(1,6 \%)$. Conformément à l'enquête Santé Québec, on note que les femmes sont plus nombreuses que les hommes à consulter pour des besoins psychologiques $(67,3 \%$ de femmes et $32,7 \%$ d'hommes). Notre enquête montre, par ailleurs, que les individus qui font appel à ces ressources appartiennent surtout au groupe d'âge des 25 à 44 ans et vivent en milieu urbain ( $10,7 \%$ à Montréal et $5,3 \%$ pour les régions autres que Québec et Montréal). Le taux de consultation des 
allophones est deux fois moindre que celui des francophones ou des anglophones. Enfin, l'analyse ne révèle aucune différence significative entre les divers niveaux de revenus.

\section{Perception du système par la population}

Dans l'ensemble, les répondants sont très positifs quant à la qualité du système de santé puisque $79,4 \%$ sont d'accord avec l'opinion que "le système de santé que nous avons au Québec est I'un des meilleurs au monde ». Une série de questions portant sur l'importance accordée aux besoins de certains groupes cibles pondérait cependant cette attitude globale face au système de santé. Interrogés sur l'accessibilité à des places en centre d'accueil pour une personne âgée, $52,5 \%$ des répondants affirment qu'elle est très restreinte et $36,9 \%$ qu'elle est plutôt restreinte. Dans l'ensemble, on considère que le système de santé répond très bien aux besoins des jeunes adultes $(79,7 \%)$, ainsi qu'à ceux des personnes handicapées $(76,1 \%)$. Par contre, plus de la moitié de la population considère que le gouvernement n'aide pas suffisamment les chefs de famille monoparentale. Résultat paradoxal dans le cadre d'un système de santé gratuit et accessible à tous, $48 \%$ des répondants considèrent que " pour les Québécois qui n'ont pas d'argent, il est très difficile d'obtenir tous les traitements nécessaires pour guérir ».

\section{Services produits par la population}

Une proportion importante de Québécois $(26,9 \%)$ affirment pratiquer une activité bénévole. De ceux-ci, 15,2\% œuvrent auprès des enfants et des adolescents, $14,1 \%$ auprès des personnes âgées, $6,4 \%$ auprès de personnes handicapées et $8,2 \%$ auprès d'autres groupes. Les bénévoles comptent à peu près autant $\mathrm{d}^{\prime}$ hommes que de femmes et autant de personnes actives sur le marché du travail que de personnes sans emploi. Par contre, les bénévoles sont relativement plus nombreux chez les personnes de 35 à 44 ans $(33,6 \%)$, chez les plus scolarisés $(33,7 \%$ ) et chez les anglophones (38,0\% contre $27,6 \%$ chez les francophones et $11,3 \%$ chez les allophones). L'action bénévole occupe donc une place relativement importante, parallèle à la couverture étatique au plan sociosanitaire.

\section{Assister un parent âgé en perte d'autonomie}

Pour diverses raisons démographiques, financières et sociales, l'assistance aux personnes âgées en perte d'autonomie provenant du 
réseau informel est une ressource extrêmement importante, sur laquelle les décideurs politiques et les planificateurs de soins et services sociosanitaires voudraient pouvoir compter. De nombreuses recherches sur l'assistance du milieu naturel aux personnes âgées en perte d'autonomie ont été réalisées, principalement aux États-Unis et en Europe. Cependant, au moment des travaux de la Commission Rochon, aucune enquête d'envergure ne permettait de saisir l'ampleur du phénomène au Québec ni les modalités et les difficultés associées à cette assistance. Aussi, notre équipe s'est attachée à faire le portrait des aidants naturels et des personnes qu'ils aident, à décrire l'assistance fournie et à identifier les difficultés associées à la situation d'aidant. La stratégie mise au point pour construire un échantillon représentatif des aidants naturels de personnes âgées en perte d'autonomie $(n=294)$ reposait sur des critères précis d'admissibilité pour les aidants, comme pour les personnes âgées déclarées en perte d'autonomie par le répondant ${ }^{2}$.

\section{Portrait des aidants et des aidés}

Parmi I'ensemble des Québécois, 4,6\% aident une personne âgée en perte d'autonomie : $2,7 \%$ aident un parent vivant à domicile et $1,9 \%$, un parent vivant en institution. Le profil sociodémographique des aidants ressemble à celui des non-aidants, sauf en ce qui concerne l'âge : les aidants sont en moyenne plus âgés ( 42,6 ans) que les non-aidants ( 39,3 ans). Par ailleurs, les femmes constituent $61,2 \%$ des aidants. Parmi les aidants, 8,7 \% aident leur conjoint ou conjointe, $55,9 \%$, leur père, mère, beau-père ou belle-mère, et $34,8 \%$ assistent un autre membre de la famille (grand-parent, frère, sœur, beau-frère, belle-sœur).

Par ailleurs, le cadre de vie des personnes aidées varie : $58,8 \%$ vivent à domicile et $41,2 \%$, en institution. Parmi les individus à domicile, $27,7 \%$ vivent seuls, $25,2 \%$ avec l'aidant, et $47,1 \%$ avec quelqu'un d'autre. Concernant les personnes en institution, la répartition est la suivante : $67,2 \%$ en centre d'accueil, $28,0 \%$ en centre hospitalier, et $4,8 \%$ en famille d'accueil.

\section{Assistance fournie}

L'assistance prodiguée par les aidants est variée : travaux ménagers lourds et légers, accompagnement, aide à la préparation des repas, aide pour l'hygiène, aide pour les déplacements intérieurs, soins thérapeutiques. À l'exception des soins médicaux et infirmiers, les besoins des personnes âgées à domicile sont presque exclusi- 
vement assurés par le répondant et la famille de l'aidé. Pour les besoins dits essentiels (alimentation, élimination, hygiène corporelle, soins thérapeutiques), l'aide provient à $61 \%$ du répondant (ou du conjoint du répondant) et à $41 \%$ des autres membres de la famille de l'aidé. L'implication du CLSC, des groupes communautaires, des voisins, des amis et du secteur privé s'est révélée minime sur ce plan. Aider un parent vivant en institution signifie surtout l'accompagner pour sortir, lui tenir compagnie, participer aux activités organisées dans son milieu de vie ou gérer ses affaires financières. Les besoins essentiels de la personne âgée en institution sont généralement comblés par le personnel de l'institution.

\section{Difficultés vécues par les aidants}

Les travaux sur l'assistance du réseau naturel concluent que les difficultés vécues par les aidants de personnes âgées en perte d'autonomie sont multiples tant au plan financier que social, physique ou psychologique. Les aidants et les non-aidants de notre échantillon décrivent leur état de santé de façon semblable. L'examen de cette dernière variable en fonction de la situation de la personne âgée montre toutefois que $11,5 \%$ des aidants de personnes âgées vivant à domicile se déclarent en mauvaise ou très mauvaise santé, alors que c'est le cas de seulement 2,2\% des aidants de personnes âgées vivant en institution. Les premiers sont également plus nombreux que les aidants d'une personne âgée en institution à déclarer s'être sentis malades au cours du dernier mois (aidant d'une personne en institution : $15,2 \%$; aidant d'une personne à domicile : $22,4 \%$ ).

Quel que soit leur niveau de revenu, la moitié des aidants de parents à domicile $(47,3 \%)$ ou en institution $(44,8 \%)$ rapportent que leur situation d'aidant entraîne des conséquences négatives sur leur vie personnelle et familiale : problèmes familiaux et réduction du temps pouvant être consacré au conjoint, aux enfants et aux temps libres. La situation des aidants de parents en centre d'accueil parait moins difficile : seuls $35,5 \%$ mentionnent ce genre de difficultés. Le concours de l'État par du soutien financier, de l'information, du gardiennage, de même que la contribution des autres membres de la famille sont mentionnés comme moyens d'alléger la tâche de l'aidant au plan physique. Les problèmes psychologiques apparaissent plus importants que les difficultés physiques : en effet, $40,9 \%$ des aidants de personnes âgées à domicile trouvent leur situation difficile au plan psychologique, alors que seulement $22 \%$ la jugent difficile au plan physique. L'assistance à un parent âgé se répercute également sur la vie professionnelle des aidants : $7,1 \%$ ont dû cesser de travailler, 
$7,0 \%$ ont dû refuser des responsabilités à leur travail et $11,3 \%$ ont dû diminuer leurs heures de travail rémunéré.

Nous avons effectué récemment une analyse secondaire de nos données (Jutras et Veilleux, 1989) qui montre que plusieurs facteurs influencent le fardeau ressenti par l'aidant : le degré d'assistance fournie, l'assistance pour les activités de la vie quotidienne, le fait de faire des démarches auprès des services officiels, le degré d'autonomie de la personne âgée et son état de santé, de même que l'âge de l'aidant et son degré de proximité avec l'aidé, le niveau de la responsabilité assumée par l'aidant, la cohabitation et le degré d'assistance provenant d'autres membres du réseau personnel.

\section{Absence d'alternative}

À l'heure actuelle, lorsqu'une personne âgée manifeste des signes croissants de perte d'autonomie, vers qui peut-elle se tourner? L'hébergement public n'est disponible en pratique que si l'état de santé est très mauvais et les ressources des programmes de maintien à domicile sont des plus limitées. D'après les résultats de I'enquête, le milieu communautaire ne fournit pas d'assistance aux personnes âgées en perte d'autonomie. Les ressources du secteur privé (par exemple : services de maintien à domicile, résidences privées pour personnes âgées) se sont récemment développées, offrant d'autres solutions; cependant, elles sont encore limitées et s'adressent à une clientèle capable d'en assumer les coûts. En principe, les ressources de maintien à domicile devraient permettre la présence de plusieurs intervenants, de divers types de solutions; en pratique, cette coexistence n'a pas été décelée auprès des personnes âgées aidées par leur famille. Les soins et services aux personnes âgées en perte d'autonomie reposent donc essentiellement sur l'un ou l'autre type de ressources : la famille, s'il s'agit d'une personne à domicile, ou le milieu professionnel, dans le cas d'un parent institutionnalisé.

\section{Assumer les responsabilités de chef de famille monoparentale}

Le nombre croissant de familles monoparentales, de même que les problèmes particuliers auxquels elles sont confrontées avaient retenu l'attention des commissaires qui voyaient dans la monoparentalité un bon cas type pour étudier les comportements de recours en matière de services sociaux définis au sens large. Dans ce volet, les 
objectifs étaient de cerner la réalité socio-économique des chefs de famille monoparentale, de décrire comment ils arrivent à résoudre leurs problèmes et, finalement, de définir leurs principales sources d'aide. Dans cette recherche, une famille monoparentale est composée d'un adulte qui vit avec un ou plusieurs enfants dont au moins un a moins de 18 ans. La situation de monoparentalité doit perdurer depuis un minimum de six mois (afin d'éliminer les biais de réponses pouvant être causés par les problèmes émotifs d'un événement trop récent et aussi afin de s'assurer de la stabilité de la situation) et ne pas être antérieure à $1969^{3}$. L'échantillon est composé de 301 chefs de famille monoparentale, âgés de 18 ans et plus. Dans notre étude, nous avons distingué trois types de situations monoparentales, selon l'événement déclencheur : le veuvage, la naissance d'un enfant en dehors de la cohabitation des parents, la séparation ou le divorce (l'union pouvant être légale ou de fait).

\section{Réalité socio-économique des chefs de famille monoparentale}

Près de neuf fois sur dix, le chef de famille monoparentale est une femme ${ }^{4}$ et si l'on compare sa situation à celle des chefs de famille biparentale, on constate qu'elle est moins scolarisée $(71,3 \%$ ont moins de 13 ans de scolarité contre $64,2 \%$ chez les autres) et touche un revenu deux fois moins élevé (17 $420 \$$ contre $39810 \$$ ). De nombreuses mères vivent de prestations de l'aide sociale : quatre sur cinq dans le cas des mères célibataires, deux sur cinq chez les séparées ou divorcées, et $13 \%$ des veuves.

Parce que ces familles accusent généralement une baisse de revenu (nous verrons plus loin que l'aide financière du père est peu fréquente), le logement devient l'un des problèmes majeurs. Près de la moitié d'entre elles $(47 \%)$ affirment avoir dû déménager à la suite du changement de statut familial et, pour plusieurs $(33 \%)$, cela a correspondu au passage du statut de propriétaire à celui de locataire.

Les femmes, plus que les hommes, ont connu des bouleversements dans leur situation de travail. Chez celles qui occupaient un emploi, près de $20 \%$ ont dû cesser de travailler au moment du changement de statut familial, alors que plus de la moitié des femmes à la maison déclarent avoir cherché du travail à ce même moment, mais seulement le tiers d'entre elles ont pu trouver un emploi. Plus de six mois après l'établissement de la monoparentalité, moins nombreuses sont les femmes à tenir maison ou à travailler à l'extérieur; par contre, le nombre de celles qui poursuivent des études ou se retrouvent en chômage est plus considérable. 
La lourdeur des responsabilités et la précarité des ressources imparties à ces chefs de famille expliquent probablement pourquoi près de la moitié d'entre elles n'ont pu prendre de vacances à l'extérieur de la maison depuis qu'elles sont seules avec leurs enfants.

\section{Problèmes et solutions}

Les chefs de famille séparées ou divorcées vivent des relations difficiles avec les pères au moment de la rupture et par la suite. Des négociations, des discussions s'entament, conduisant parfois à des refus de collaboration, parfois à un support plus ou moins tangible de la part du père et parfois aussi à des situations de violence.

Le tiers des mères célibataires ont conservé des liens avec le père et une très faible proportion en reçoivent une aide quelconque. Quant aux mères séparées ou divorcées, plus de la moitié (56 \%) ont demandé une pension alimentaire pour les enfants; parmi celles-ci, près de deux mères sur cinq ont obtenu un jugement sanctionné par la cour, et une sur cinq a pris entente directement avec l'ex-conjoint. Cependant, le tiers de celles qui devaient recevoir une pension ne l'ont pas obtenue. En bref, plus de la moitié des mères séparées et divorcées et presque toutes les mères célibataires ne reçoivent aucune aide financière du père.

La majorité des parents (79\%) s'étaient entendus sur les droits de visites, cependant une fois sur deux on constate la présence d'un différend entre les ex-conjoints sur le sujet. De plus, près d'une femme sur trois, chef de famille monoparentale, séparée, divorcée ou célibataire, affirme que le père de l'enfant a eu, au cours de la période entourant la séparation, des comportements plutôt violents, généralement dirigés vers la mère.

De nombreuses difficultés assaillent les chefs de famille monoparentale concernant l'organisation financière et matérielle, les responsabilités familiales, les problèmes conjugaux et personnels, etc. Pour ce faire, elles ont recours à deux catégories principales de ressources, l'entourage et les services de l'État.

Avant la rupture, près du quart des séparées et divorcées ont suivi une thérapie conjugale ou ont fait appel à un conseiller matrimonial ou à un service de médiation. Dans les cas de violence domestique, quelques femmes se sont réfugiées dans une maison d'hébergement ou à l'hôpital, d'autres ont fait appel à la police ou à des amis.

Seulement le tiers des chefs de famille monoparentale déclarent avoir reçu de l'aide pour le soin et l'éducation des enfants; cette aide provient d'un membre de la famille (66\% des cas), d'amis $(24 \%)$ ou du père de l'enfant (22\%). Quand ce dernier ne paie pas la pension 
alimentaire convenue, les femmes font appel à la médiation (13\%), se tournent vers le service de perception de pensions alimentaires instauré par l'État ou encore manifestent l'intention de le faire (36\%).

Des problèmes de santé affectant les enfants sont apparus dans plus de $30 \%$ des cas à la suite de la monoparentalité; toutefois, selon les mères, seulement $10 \%$ seraient directement attribuables à la séparation. La plupart des problèmes signalés sont des problèmes psychologiques ou de comportement. Dans la majorité des cas, l'aide reçue vient encore une fois de la famille, alors que la mère souhaiterait recevoir de l'aide du père de l'enfant.

Par ailleurs, un peu moins de la moitié des chefs de famille déclarent avoir vécu des problèmes personnels depuis l'événement déclencheur, mais seulement une femme sur six y verrait un lien direct avec la situation de monoparentalité ou la séparation. Tout comme pour les enfants, ces problèmes sont reliés à leur santé physique et psychique. L'aide reçue provient de la famille dans la très grande majorité des cas, mais aussi des amis et des professionnels de la santé et des services sociaux. D'autre part, à une question plus globale sur l'aide reçue, plus de la moitié $(55 \%)$ des chefs de famille monoparentale désignent une relation avec d'autres parents qui élèvent seuls leurs enfants : les deux tiers ont reçu d'eux un soutien moral.

Dans l'ensemble, plus des deux tiers des chefs de famille monoparentale reçoivent de l'aide de leur entourage et cette aide augmente en fonction du niveau de besoins, les plus démunies d'entre elles déclarant un pourcentage plus élevé. Par contre, seulement $13 \%$ des chefs de famille ont reçu de l'aide de groupes communautaires alors qu'un autre $10 \%$ auraient souhaité en recevoir.

Si l'on se tourne du côté des services institutionnalisés, les chefs de famille monoparentale ont recours aux services de santé dans la même proportion que la population en général. Par contre, leur utilisation de services sociaux est trois fois plus fréquente $(9 \%$ contre $3 \%$ ). Leur estimation des services offerts est toutefois moins bonne que celle exprimée par l'ensemble de la population : elles se révèlent moins satisfaites des services de santé $(71 \%$ contre $80 \%)$, ont plus de plaintes à formuler à l'égard des services sociosanitaires ( $11 \%$ contre $5 \%$ ) et expriment un plus grand désaccord avec l'opinion que "le gouvernement aide suffisamment les chefs de famille monoparentale » $(78 \%$ contre $53 \%)$.

\section{Principales sources d'aide}

Malgré des gains évidents du côté de certaines législations entourant les ruptures d'unions, la garde des enfants et la perception 
de la pension alimentaire, les chefs de famille monoparentale séparées ou divorcées continuent, dans l'ensemble, d'assumer principalement les charges financières et éducatives des enfants. II n'est donc pas surprenant de les voir se tourner vers leur entourage et vers les services de l'État dans un grand nombre de cas. Compte tenu de la nature des besoins exprimés et des problèmes rencontrés, c'està-dire ressources matérielles, garde des enfants, problèmes d'ordre psychologique, l'aide vient beaucoup plus de l'entourage, c'est-à-dire de la famille et des amis, que des services institutionnalisés. Rien d'étonnant à cela si I'on sait que le système sociosanitaire du Québec est principalement orienté vers les soins médicaux d'ordre curatif. De façon générale, les chefs de famille monoparentale disent avoir à peine plus de problèmes de santé que la moyenne de la population et ne recourent pas plus aux services de santé que l'ensemble des gens. II en est tout autrement des problèmes d'ordre psychosocial puisqu'elles font appel aux services sociaux trois fois plus souvent que la moyenne. Par ailleurs, elles se révèlent beaucoup plus insatisfaites que l'ensemble de la population, des services sociaux et sanitaires offerts. Ces résultats suggèrent que des améliorations pourraient être apportées aux ressources gouvernementales, notamment au plan du soutien financier aux familles monoparentales, du versement des pensions alimentaires ou des ressources contre la violence conjugale.

\section{Conclusion}

Au-delà de l'éclairage particulier jeté sur les problématiques étudiées, notre enquête permet de dégager un certain nombre de constats sur le rapport aux services sociaux, qui diffère nettement du rapport aux services de santé. L'existence d'un système intégré de soins et de services, gratuit et accessible à tous les Québécois, ne signifie évidemment pas que tous les problèmes sociaux et de santé sont pris en charge par l'État. Ceci est particulièrement vrai pour les problèmes sociaux dont la variété, l'ampleur et la complexité entraînent une multiplication des services dans les secteurs communautaire et privé, de même que l'utilisation importante des ressources venant des réseaux naturels d'entraide.

Confrontés à un problème de santé, les gens se tournent habituellement vers les ressources de l'État : le médecin, l'urgence et le CLSC. Ce sont là des ressources gratuites, facilement identifiables et relativement accessibles, et que les gens considèrent, de façon générale, aptes à résoudre ou du moins à atténuer leur problème. Cependant, lorsque le problème est d'ordre social, ou psychosocial, la situation est différente. D'abord l'accessibilité aux ressources institutionnelles devient moins évidente. De surcroît, les problèmes 
"sociaux " font encore partie du domaine de la vie privée et bien souvent le réseau naturel d'entraide sera alors préféré à tout autre. Dans plusieurs de ces cas, il n'est pas toujours facile de départager ce qui relève du manque de ressources institutionnelles ou communautaires spécifiques, ou du choix de la personne ayant besoin d'aide.

Les résultats de l'enquête montrent que la famille et l'entourage continuent, comme par le passé, à assurer un soutien et une aide de première instance dans bien des domaines. Pourront-ils maintenir ce rôle à l'avenir ? On a vu que la responsabilité d'aidant naturel est souvent lourde à porter. Les services du système actuel ont été conçus sur le modèle des besoins de santé individuels, c'est-à-dire en fonction des individus et non des familles; pourtant tout semble indiquer que les besoins de ces dernières iront en croissant. Ainsi, les décideurs seront de plus en plus confrontés aux besoins causés par l'augmentation du nombre de personnes âgées et du nombre de familles monoparentales. Jusqu'à maintenant, le réseau naturel pouvait suppléer aux services étatiques ou les compléter, mais sera-ce le cas avec les changements que connaît la famille (divorce, présence massive au foyer des jeunes de 18-25 ans, dénatalité, investissement professionnel des femmes, etc.) ? Si l'on veut favoriser l'assistance provenant du réseau naturel, ne faut-il pas prévoir une réorientation des services ou du soutien accordé aux familles et aux groupes communautaires?

Le système institutionnel québécois donne priorité au recouvrement de la santé et cette partie de son mandat rejoint l'assentiment de la population qui se montre satisfaite des services offerts. Toutefois, la satisfaction est moindre lorsqu'il est question de problèmes sociaux ou psychosociaux : par exemple, la majorité de la population déclare que l'État n'apporte pas une aide suffisante aux familles monoparentales. Si celui-ci ne réajuste pas ses priorités, des segments de plus en plus importants de la population risquent d'être négligés. II s'agit d'un défi important : I'expansion des services sociaux et psychosociaux entraîne une inévitable augmentation des coûts, donc du fardeau fiscal. Par contre, sans un tel développement, la famille risque de devoir augmenter significativement la part de services qu'elle doit assumer, sans pour autant être en mesure de répondre aux attentes dirigées vers elle.

L'augmentation prévue du nombre de personnes âgées et des problèmes d'ordre psychosocial en général laisse entrevoir une augmentation des besoins, en particulier dans le domaine social et sociosanitaire. Les ressources communautaires, pour dynamiques qu'elles soient, n'ont occupé jusqu'à maintenant qu'un territoire restreint; elles pourraient à l'avenir jouer un rôle plus important en raison même de leur polyvalence et de leur capacité d'innovation en 
ce qui concerne les pratiques et les approches thérapeutiques et d'assistance. II faudra alors consentir des mécanismes de support financier plus adéquats pour ces ressources, tout comme il faudra mettre en place des mesures de soutien économique et organisationnel pour aider les familles fournissant assistance aux proches.

Ce que révélait notre enquête en 1987 est toujours d'actualité en 1990. À l'aube d'une nouvelle réforme, la réorganisation prévue des services de santé et des services sociaux viendra-t-elle changer les paramètres du recours aux services sociaux ? La réorganisation des services sociosanitaires prendra-t-elle en compte le potentiel réel d'assistance des familles auprès de leurs parents âgés ? Saura-t-on prévoir des modalités d'assistance variées pour aider les familles monoparentales aux prises avec diverses difficultés? Comment devra-t-on définir les rôles et fonctions des professionnels du réseau ? Se donnera-t-on les moyens d'atteindre les objectifs de bien-être décrits dans le document d'Orientations (Ministère de la Santé et des Services sociaux, 1989) ?

Que l'on examine le réseau public, les organismes communautaires ou les ressources personnelles des individus et des familles, il est clair que les "services sociaux" constituent un univers en transition. En effet, les problèmes sociaux se transforment, les ressources du réseau personnel se trouvent tantôt davantage sollicitées, tantôt moins présentes, les ressources communautaires continuent à se développer, élargissant leur base et leur rayon d'action, alors que l'État cherche à rationaliser, sinon à diminuer ses dépenses. Au fond, ce qui se profile, à l'aube des années 1990, c'est une recherche de nouvelles formes de solidarité sociale, à la fois pour faire face aux problèmes sociaux que nous a légués le passé et aux problèmes en émergence pour l'avenir.

\section{Notes}

* Note des auteurs. Cet article repose sur les résultats de la recherche que nous avons menée pour la Commission d'enquête sur les services de santé et les services sociaux, en collaboration avec Pierre Bouchard, Renée B.Dandurand et Louise Guyon. Nous remercions le ministère de la Santé et des Services sociaux, le Conseil québécois de la recherche sociale, de même que le Conseil de recherches en sciences humaines du Canada de leur appui financier à diverses étapes de nos travaux. Prière d'adresser toute correspondance à $\mathrm{S}$. Jutras, Groupe de recherche sur les aspects sociaux de la prévention (GRASP), Université de Montréal, 2801 Édouard-Montpetit, bureau 162, Montréal, H3T 1 J6.

${ }^{1}$ Seules les données essentielles à la compréhension du présent texte sont rapportées ici; le lecteur intéressé à plus de détails est invité à consulter Renaud, Jutras et Bouchard (1987). 
${ }^{2}$ Cette stratégie est décrite en détail par Renaud, Jutras et Bouchard (1987).

${ }^{3}$ Des modifications législatives canadiennes rendaient alors le divorce plus accessible aux Québécois.

${ }^{4}$ Compte tenu de cette réalité et pour alléger la lecture de cette partie du texte, nous avons choisi de traiter l'expression " chef de famille monoparentale " au féminin, ce qui inclut les femmes et les hommes.

\section{Références}

GrandmaISON, A. (1981). Les nouveaux bénéficiaires du CSSMM : caractéristiques individuelles. (Dossier clientèles, document $\mathrm{n}^{\circ} 2$ ). Montréal : CSSMM, Direction des services professionnels, Service de la recherche.

Jutras, S. et F. Veilleux, avec la collaboration de M. Renaud (1989). Des "partenaires" méconnus: les aidants des personnes âgées en perte d'autonomie. Montréal : GRASP, Université de Montréal.

Ministère de la Santé et des Services sociaux (1989). Orientations. Pour améliorer la santé et le bien-être au Québec. Québec : Ministère de la Santé et des Services sociaux.

Renaud, M., S. Jutras et P. Bouchard, avec la collaboration de L. Guyon et R. B.-DANDURAND (1987). Les solutions qu'apportent les Québécois à leurs problèmes sociaux et sanitaires. Trois cas types : s'occuper d'un parent âgé, soulager son mal de dos, être chef de famille monoparentale. Québec : Les Publications du Québec. 